\title{
Abstracts from National Perinatal Association 2020 Conference, December 2-4, 2020: New Framework for Multidisciplinary Care in the $4^{\text {th }}$ Trimester
}

Jerasimos (Jerry) Ballas, MD, MPH, FACOG

The National Perinatal Association (NPA)is an interdisciplinary organization that strives to be a leading voice for perinatal care in the United States. Our diverse membership is comprised of healthcare providers, parents \& caregivers, educators, and service providers, all driven by their desire to give voice to and support babies and families at risk across the country.

Members of the NPA write a regular peer-reviewed column in Neonatology Today.

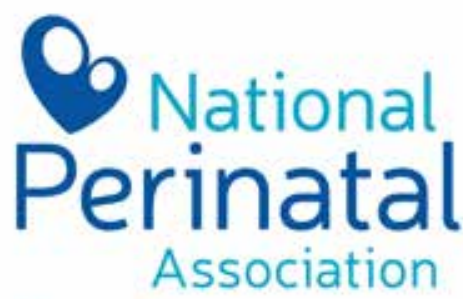

Educate. Advocate. Integrate.

"This year the chosen

theme focused on the 4th

Trimester and the various

areas of vulnerability for

parents and infants during

this time, as well as the

resources available for

support."

Readers can also follow

via our Twitter Feed to practice, and articulate standards and competencies from which to standardize the practice of all members of the interprofessional collaborative team in the intensive care units. The six areas include systems thinking, positioning and touch for the newborn, sleep and arousal interventions for the newborn, skin-to-skin contact with intimate family members, reducing and managing pain and stress in newborns and families, and the management of feeding, eating and nutrition delivery of the baby. Professional and parent participants attending three Gravens meetings provided feedback to the consensus committee and an expert panel of interprofessionals also provided recommendations. An overview of this work has been published, and the standards and competencies are available on line.

Lessons learned: Currently there is no available standardization of developmental, family centered care for interprofessional use. The panel of leaders in the field worked collaboratively to examine the literature and produce well documented standards and competencies for practice in intensive care. Further work needs to support the implementation of the standards, competencies and best practices of IFCDC by the interprofessional collaborative health team in the hospital setting.

Implications for practice: The publication of these standards and competencies will be the first available empirically based interprofessional expectations for developmental care. To the extent that they can be readily implemented they will provide a national impact on developmental outcomes for babies and their families. Recommendations for inclusion of families, and transition of the baby and family from the hospital to communities, are infused throughout the document and should provide continuity for service provision from hospital to home.

\section{NPA2020-2}

Comprehensive postpartum care: Assessment 
of varying provider practices and patient experiences.

Authors: Alanna M. Cruz-Bendezú, BA, (acruzbendezu@gwu.edu), Nicole Gunawansa, BS*,Arlin Delgado, MD**, Jenna Wade, BA*, Arianna Prince, BS*, Michael Power, PhD***, Jay Schulkin, PhD****, Charles Macri, MD*

\section{Affiliations:}

*The George Washington University School of Medicine and Health Sciences, Department of Obstetrics and Gynecology at the George Washington University School of Medicine and Health Sciences, 2150 Pennsylvania Avenue, NW Suite 6A, Washington, DC. 20037

** The University of South Florida Morsani College of Medicine, Department of Obstetrics and Gynecology, 2 Tampa General Circle, Tampa, FL 33606

*** Smithsonian National Zoological Park and Conservation Biology Institute, 3001 Connecticut Ave NW, Washington DC 20008

**** The University of Washington, School of Medicine, Department of Obstetrics and Gynecology, 1959 NE Pacific St Seattle, WA 98195-6460

Introduction: Women experience challenges that affect their health and their ability to care for their infant during the postpartum period. Up to $40 \%$ of women do not attend the initial postpartum visit. We investigated how different types of providers manage postpartum education and assess current patients' concerns and challenges of the postpartum period. A prenatal assessment that identifies postpartum concerns could help providers develop individualized care plans that improve postpartum care.

Methods: Patients at a major urban OB/GYN clinic were recruited for an IRB-waived voluntary survey. Descriptive statistics, chi-squared tests, and odds ratios were used for analysis.

Results: Among the 250 women in their $3^{\text {rd }}$ trimester, there were high levels of concern regarding breastfeeding (59.2\%), experiencing "baby blues" (50.0\%), losing pregnancy weight (50\%), tiredness $(64.0 \%)$, and pain after birth (60.0\%). However, only $52.4 \%$ reported discussing plans to feed their baby postpartum with even less discussion on other important postpartum topics such as challenges they might experience (30.0\%), physical activity (20.4\%), and losing pregnancy weight (12.0\%). Reported discussions regarding postpartum care by type of provider were statistically significant, with midwives less likely to discuss a postpartum care plan than medical doctors (OR .10; 95\% Cl 0.05-0.20; P<.001). However, patients reported midwives were as likely to discuss postpartum challenges $(P=.9565)$.

Conclusion: Patients expressed postpartum concerns but less than half report discussions with providers on aforementioned topics. The likelihood of reported discussions regarding postpartum care varied by type of medical provider, which highlights the potential benefits of multidisciplinary collaboration. We suggest that a standard $3^{\text {rd }}$ trimester survey might improve postpartum care plans.

\section{NPA2020-3}

Helping Parents When the $4^{\text {th }}$ Trimester is in the NICU- An Integrated Training Model for NICU Physicians

\section{INNOVATIVE MODELS OF CARE}

Background: Nearly all parents whose babies require NICU care experience some level of distress, with up to $20-60 \%$ developing postpartum depression, anxiety, or posttraumatic stress disorder. These conditions adversely impact parent-infant attachment and overall parenting behaviors, leading to higher risks of worse physical and developmental outcomes in the babies. Research suggests that providing psychosocial support to NICU parents can reduce their distress, depression, anxiety, and increase the possibility of the parent-infant bonding and attachment. Therefore, providing psychological care to families in the NICU may lead to overall healthier infant outcomes. However, it has been noted that many pediatric and neonatology trainees, and neonatologists, feel they do not have the self-efficacy care for distressed and anxious parents. In 2014, the American Board of Pediatrics Strategic Planning Committee identified the areas of behavioral and mental health as the highest priorities for education of pediatric trainees. This led to the development of the Roadmap Project, which advocates supporting "the resilience, emotional, and mental health of pediatric patients with chronic conditions and their families." While some neonatology fellowship programs teach communication skills for high stress situations, no comprehensive program exists in psychosocial care of NICU families. We have created the first such course for this purpose, in alignment with the Roadmap's Key Drivers. This poster will discuss the development and piloting process of this training program.

Action: This is a prospective an educational intervention on neonatology fellows in the United States. All accredited neonatology fellowship programs have been contacted for possible enrollment of their fellows in the study. Consenting fellows complete, at a minimum, all portions of the online program including both assessments of self-efficacy and knowledge at all time points. Fellowship programs have the option to have their fellows participate in the evaluation of clinical fellow skill via parent evaluation. Fellows who are local to the children's hospital that holds the institutional review board approval for this study have been offered participation in simulated parent conversations that require prac- tical application of the concepts found in the course. There are 27 available fellows considering participation in the simulation.

Enrolled fellows are given access to a 4-module online course covering the topics of Recognizing and Mitigating Parental Emotional Distress, Infant Distress, Communication, and Developmental Care. This course was modified specifically for education of neonatal fellows from a course already offered to NICU staff, called "Caring for Babies and Their Families: Providing Psychosocial Support in the NICU". The course has its foundation in the "Interdisciplinary Recommendations for Psychosocial Support of NICU Parents," as well as in the concepts of trauma-informed care. It is available at www.mynicunetwork.com.

A subgroup of fellows will go through a simulation session at an immersive learning center that has extensive experience in physician training via simulation, including simulations of emotional distress in the medical setting. The center will provide training of our selected simulated patients in conjunction with study team to ensure alignment with study goals. Simulated patients/parents will go through a minimum of 2 days of training on study scenarios. The fellows will each interact with a simulated patient representing a NICU parent confronting an "everyday" situation, as opposed to a situation requiring delivery of "bad news." Scenarios will be videotaped for later review. Fellows participate in these sessions for a half-day, personally perform in one scenario and watch scenarios of 2 other fellows. Groups of 3 fellows will participate in debriefings using video tape after each scenario.

Lessons Learned: This poster will discuss lessons learned from the development of the training program and provide highlights of program content. Additionally, through meetings with the identified field representatives during interdisciplinary collaborations between parents, neonatology, psychiatry, and psychology the authors will share topical insights regarding the teaching of mitigating both parental and infant distress for trainees. Topics include providing culturally sensitive care in the NICU, psychological impact of trauma on babies and their families, and effective communication strategies in the NICU.

Implications for Practice: The time is now to focus our efforts heavily on the fourth trimester. For parents who have an infant in the NICU, the fourth trimester comes way too soon and increases the potential for needed psychological support. Our project hopes to address a high priority educational need as identified by the American Board of Pediatrics and the Accreditation Council for Graduate Medical Education. A new core program requirement became active July 1, 2019 for pediatric training programs to develop curricula to train residents and fellows on screening for mental health issues in their patients and in the case of infants, in their parents. No national programs 
2020 SONPM Virtual Awards

\section{Ceremony}

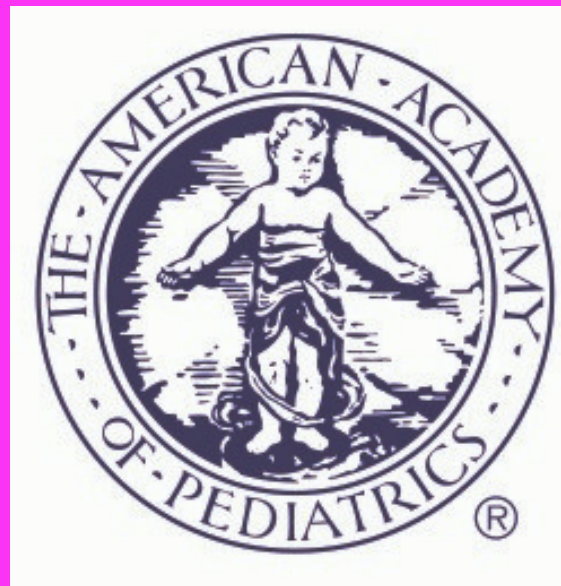

Thursday

April 22, 2021

12:30PM PT

1:30PM MT

2:30PM CT

3:30PM ET

**Sponsored By** Abbott Nutrition \& Mead Johnson Nutrition

\section{Join Ceremony Here}

Password: 0422

2:30PM- Intro \& Update from the Chair

2:40PM- Intro to Merenstein Lecture-

2020 Merenstein Lecture: "Moral Status

and Justice Considerations in the NICU"

Mark Mercurio, MD, FAAP

Sponsored by Abbott Nutrition

3:05PM- Intro to 2020 Fanaroff Education Award-

2020 Education awardee- Bill Benitz, MD, FAAP

Sponsored by Mead Johnson Nutrition

3:15 PM- Intro to 2020 Landmark Award-

2020 Landmark awardee - Tom Wiswell, MD, FAAP Sponsored by Mead Johnson Nutrition

3:25PM- Intro to Thomas Cone History Lecture-

2020 Cone History Lecture: "Seventy-five years of

Progress in Neonatal Sepsis: The Cha-cha Hypothesis" Rich Polin, MD, FAAP

Sponsored by Abbott Nutrition

3:50PM- Intro to 2020 Virginia Apgar Award-

2020 Apgar awardee- Betty Vohr, MD, FAAP

Sponsored by Abbott Nutrition

\section{4:05PM- Closing}

Presenters: Lily Lou, MD, FAAP; Mark Hudak, MD, FAAP;

David Stevenson, MD, FAAP; Bill OH, MD, FAAP

\section{\#SONPM2020awards}

Password: 0422 
exist for addressing this important topic, leaving programs to find local resources and craft individual and less comprehensive training. Our project could provide an example to other pediatric training programs. If found to be effective, our course, or elements of our course, could be adapted for the training of residents and fellows in other pediatric subspecialties.

Our project has the potential to impact thousands of NICU families at a crucial time for the development of their foundational relationships with their infants. High rates of distress have been documented in NICU families making the impact of trainee understanding, efficacy and skill at caring for them particularly important. Distress experienced by both parents and their infant(s) in the NICU may impair both the emotional and physical health of each, as well as the family's relationships throughout childhood, creating unseen negative impacts in both populations. Finally, our training program has the ability to address a known educational deficit, possibly impact thousands of parents and their infants, and provide a model for other pediatric training programs to adapt for their specific patient populations and needs.

NPA2020-4

A Critical Analysis of Intimate Partner Violence During Pregnancy in The United States

Elizabeth Filipovich, MPH

\section{Abstract}

Introduction: Intimate partner violence during pregnancy is a significant public health problem with several associated adverse maternal and fetal outcomes, including preterm labor, low birthweight and maternal mortality. This critical analysis will explore factors that contribute to the high incidence of IPV in pregnancy, current prevention best practices, and interventions suited to reduce the incidence of IPV among pregnant women.

Methods: A literature review was performed using PubMed and George Washington University's Himmelfarb Health Sciences Library.

Results: Pregnancy is an optimal time to screen for IPV due to repeated contact with a care provider throughout a woman's pregnancy. Barriers to screening for IPV, inadequate provider education, lack of appropriate resources, and a lack of consensus regarding screening strategies and tactics contribute to lack of intervention for women who are experiencing IPV in pregnancy.

Conclusions: Progress in addressing IPV requires further research, including broad based controlled trials of intervention methods applied in diverse populations. In particular, studies comparing effectiveness of IPV intervention among various pregnant populations have the potential to determine whether the period of pregnancy presents a greater opportunity for success in reducing IPV than intervention at other times in a woman's life. Further research into the impact of IPV intervention on birth outcomes may provide critical information on which to base specialized programs of care for populations most at risk for low birth weight and preterm birth. Model programs have demonstrated effectiveness in reducing harm related to IPV using a combination of interventions. Testing these models can further the evidence base on which to build standard practices for effectively addressing this public health problem.

\section{NPA2020-5}

"Babywearing" as a Tool to Decrease Pain Associated with Neonatal Abstinence Syndrome

Introduction: Prescription opioid sales in the U.S. has almost quadrupled from
1999 to 2014; correspondingly, infants diagnosed with Neonatal Abstinence Syndrome (NAS) has increased more than fivefold. NAS is commonly associated with maternal opioid use and includes symptoms such as high-pitched crying, tremors, and poor feeding. Infants with NAS are accustomed to drug exposure in utero; consequently, when the drug is no longer present, the absence of the stimuli is painful. Elevated heart rate (HR) is synonymous with increased infant pain and stress in adults. Research on skin-to-skin or kangaroo care has found decreased perceptions of pain (i.e., HR) during heel prick procedures. The purpose of the study is to examine whether infant carrying or "babywearing" (i.e. holding an infant on one's body using cloth) can reduce stress and symptoms associated with NAS.

Methods: This repeated-measure study took place in a Neonatal Intensive Care Unit (NICU) in the Southwest USA. Starting when infants were four days old, physiological readings ( $\mathrm{N}=97$ readings; $\mathrm{N}=15$ infants; $53 \%$ White, $20 \%$ Hispanic, 13\% African American; $53 \%$ female) were assessed daily. Heart rates of infants and individuals wearing the infant (e.g. parents, nurses) were taken every 15 -seconds before- (no touching), during- (20 minutes into being worn in a carrier) and post-babywearing (five to ten minutes later), approximately a forty-five minute procedure from start to finish. A finger plethysmograph, also known as a pulse oximeter, measured heart rate for the adults wearing the infants. Infants were continuously monitored by cardiopulmonary machines using a pulse oximeter wrapped around their foot.

Results: A 3-Level Hierarchical Linear Model (HLM) was used in order to account for the nested data (HRs nested within readings, nested within infant-adult dyads) at three time points (before, during, and after babywearing). We found that

\section{Newly-Validated Online NICU Staff Education}

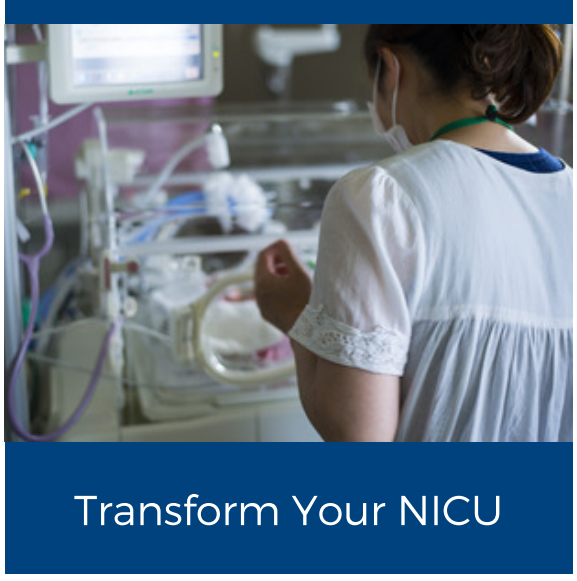

\section{Caring for Babies and their Families:} Providing Psychosocial Support to NICU Parents

\author{
based on the "Interdisciplinary Recommendations for Psychosocial \\ Support for NICU Parents."
}

Contact sara@mynicunetwork.com for more information.

\author{
Brought to you by a collaboration between \\ - National Perinatal Association \\ - Patient + Family Care \\ - Preemie Parent Alliance
}

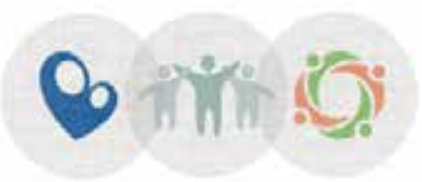

www.mynicunetwork.com 
babywearing decreased infant and caregiver heart rates. Approximately, across a 30-minute period, infants worn by parents decreased 15 beats per minute (bpm) compared to $5.5 \mathrm{bpm}$ for infants worn by an unfamiliar adult, and adults decreased by 7 bpm (parents) and nearly 3 bpm (unfamiliar adult).

Discussion: Findings from this study suggest that babywearing is a non-invasive and accessible intervention that can decrease symptoms in infants diagnosed with NAS. Babywearing is cost-effective, culturally relevant, and can be done by noncaregivers (e.g., nurses, family members, friends). Results suggest that babywearing is especially calming when parents are the ones wearing the infants. Babywearing supports parenting by including the parent in the treatment and empowering them in caring for their infant. This intervention can be used outside of the NICU and provide additional support to parents and caregivers once infants are discharged. Close physical contact, by way of babywearing, can improve infant outcomes in NICUs as an alternative to pharmacological treatment.

\section{NPA2020-6}

\section{Caring for Women and Their Families: Providing Psychosocial Support Dur- ing Maternity Care}

Authors: S Hall, A White, L Baker, A Brown, S Detlefs, ML Martin, C Milford, $S$ Wolf, K Saxer, J Ballas, K Sorrells, MA Davids, B Boet, E Thatcher, C Duffy, and T Pella.

Background: The Accreditation Council for Graduate Medical Education (ACGME) has outlined numerous milestones that residents in obstetrics/gynecology must achieve during their training. These include development of: 1-compassion, integrity, and respect for others, 2- respect for patient privacy, autonomy, patient-physician relationship, 3- interpersonal and communication skills necessary for communication with patients and families, and 4- interpersonal and communication skills necessary to provide informed consent and shared decision-making (ACGME, 2019).

We created an innovative online educational program focused on these oftenneglected areas of training for all providers of maternity care, including physicians in training (residents and fellows), practicing physicians, nurses, and other practitioners at the bedside. A key principle of our program development was that it was both interdisciplinary and interprofessional, including contributions by patients. We applied the concepts of trauma-informed care in the setting of providing maternity care as our foundation for training. We used as our exemplar an educational program we previously developed for all staff providing care in Neonatal Intensive Care Units. This program has been found to be effective at improving nurses' (the primary study population) knowledge and confidence in providing psychosocial support to NICU parents (Hall, 2019). Additionally, we wanted to satisfy the mandate passed by several states requiring that physicians who provide maternity care receive training in perinatal mental health issues.

Content/Action: A multidisciplinary and interprofessional team consisting of obstetricians, specialists in neonatal and perinatal medicine, nurse midwives, obstetric nurses, psychologists, and patients developed a 6-course online learning program that contains the following topics: 1- Using Trauma-informed Care as a Basis for Communication in Maternity Care, 2-Perinatal Mood and Anxiety Disorders: Providing Emotional Support During Maternity Care, 3- Providing Support During the Antepartum Period of Maternity Care; 4- Providing Support During the Intrapartum Period of Maternity Care, 5- Providing Support During the Postpartum (Fourth Trimester) Period of Maternity Care, and 6-Supporting Maternity Care Staff as they Support Patients. Patients contributed personal narratives to demonstrate learning points, collected resources they felt would be helpful to clinicians, and helped to review and edit all content. Each course describes how trauma-informed care can be integrated into obstetric care to ensure patients feel safe, and invested as partners in their own care at every step along the way. High risk social and emotional factors, and how to identify and respond to them, are enumerated. There are also multiple links to other sites on the internet that reinforce the content being presented, as well as downloadable documents that further enhance learning by demonstrating best practices. Courses have interactive cases to reinforce clinician learning. Each course has an extensive bibliography, and all content is firmly grounded in evidencebased literature.

Lessons Learned: An interdisciplinary and interprofessional model can be successfully used to create educational content for providers that speaks to their patients' needs. This model affords providers the opportunity to understand the patient experience from a deeper, more personal, and more meaningful perspective.

Implications for Practice: An innovative online learning program has the potential to widely disseminate educational content on providing psychosocial support, which is required in obstetric training but is not often a specific part of training curricula. Enhancing provider understanding of the patient experience can lead to increased sensitivity to patient needs and improvement in both compassion and in communication skills. Attention to staff's own needs for emotional well-being is a critical part of the curriculum, as quality care can best be delivered by providers who can avoid burnout.

\section{References:}

1. ACGME. 2019. "ACGME Program Requirements for Graduate Medical Education in Obstetrics and Gynecology." Accreditation Council for Graduate Medical Education. https:// www.acgme.org/Portals/0/PFAssets/ ProgramRequirements/220 ObstetricsAndGynecology 2019 TCC. pdf?ver=2019-04-26-111908-393.

2. Hall, SL, ME Famuyide, SN Saxton, TA Moore, S Mosher, K Sorrells, CA Milford, and J Craig. 2019. "Improving Staff Knowledge and Attitudes towards Providing Psychosocial Support to NICU Parents through an Online Education Course." Advances in Neonatal Care 19 (6): 490-99. $\quad$ https://doi.org/10.1097/ ANC.0000000000000649.

\section{NPA-2020-7}

Breastfeeding and Marijuana Use: An Ethical Analysis of Current Practice

Research Submission for National Perinatal Association 2019 Conference

Marielle S. Gross, MD, MBE1, Carla Bossano, MD2, Nadine Rosenblum, RN, IBCLC3, and Lorraine Milio, MD, MPH2

Johns Hopkins Berman Institute of Bioethics, Johns Hopkins School of Medicine, and Johns Hopkins Hospital3; Baltimore, Maryland

Introduction: U.S. guidelines recommend breastfeeding women avoid marijuana given concerns about infant neurodevelopment. Unfortunately, this has resulted in many physicians and hospitals prohibiting women who use marijuana from breastfeeding despite inconclusive evidence of harm and well-known benefits of breastfeeding. Meanwhile, marijuana use is increasing among reproductive-aged women, and complex personal/socioeconomic factors affect feeding choices. We assess evidence and ethical justification for current practice.

Methods: We review: (1) Harm to infants from breastmilk marijuana exposure vs. avoiding breastfeeding, (2) Maternal health and psychosocial considerations, and (3) 
Current practices in light of principles of beneficence, justice, and autonomy.

Results: (1) First, delta-9-tetrahydrocannabinol (THC) is excreted in breastmilk and limited data (three studies with human subjects and three animal studies) suggest neurobehavioral changes among infants whose mothers use marijuana during breastfeeding, though effects of in utero vs. breastmilk exposure are difficult to distinguish and interpretation is limited by socioeconomic and other confounders. There are also concerns that marijuana use negatively impacts safe infant care and that the average concentration of THC in marijuana has increased in the years since the relevant studies were completed. Meanwhile, avoiding breastfeeding increases infants' risk of sudden infant death syndrome, sepsis, necrotizing enterocolitis, diabetes, asthma, and obesity. Available evidence is unclear regarding whether infants who were already exposed to THC in utero would be worse off with continued exposure through breastmilk vs. with increased risks associated with formula feeding. (2) Women who do not breastfeed have increased risk of cardiovascular disease, reproductive cancers, diabetes, depression and unintended pregnancy, all major sources of morbidity and mortality for U.S. women. Marijuana use is highest among underserved minority women who disproportionately suffer from the health consequences that breastfeeding may mitigate, and who are especially vulnerable to punitive damages (e.g., criminal charges related to drug use or Child Protective Services involvement). Maternal desire to bond with her infant through breastfeeding, cultural norms, values, and social pressures, and the financial burden of formula all may exacerbate the harm of telling women to avoid breastfeeding. (3) The national recommendation that women who breastfeed should avoid marijuana is interpreted by some physicians and hospitals as a policy that women who use marijuana should not breastfeed. This practice fails to account for the risks of avoiding breastfeeding for both infants and women, possibly exaggerating the strength of available evidence about harms from breastmilk THC exposure, and thus may not optimize health outcomes. In addition to potentially exacerbating existing health disparities, this practice may be unjust if women who screen positive for THC during pregnancy are told they are 'not allowed' to breastfeed regardless of whether they are actively using at time of delivery. Given the clinical equipoise regarding the best feeding method for infants whose mothers use marijuana, particularly if they were already exposed to THC in utero, and the large range in frequency/intensity of maternal marijuana use, an individualized, shared decision-making approach is appropriate. Furthermore, a woman's autonomy may be compromised if crucial postpartum lactation support is withheld while she is in the hospital postpartum or if she is concerned that breastfeeding against recommendations may jeopardize custody of her infant.

Discussion: Ultimately, failure to account for risks of avoiding breastfeeding for infants and women, with attention to epidemiology of marijuana use and breastfeeding-associated health effects, may result in policies which do not optimize health outcomes. Restrictive breastfeeding practices for women who use marijuana which do not utilize an individualized, shared decision-making approach are neither medically sound nor ethically justified, and may disproportionately undermine the health of underserved women and infants. Unbiased, culturally-informed and evidencebased counseling would promote open patient-provider communication may improve long-term health.

\section{NPA-2020-8}

Breastfeeding and Marijuana Use: An Ethical Analysis of Current Practice

Research Submission for National Perinatal Association 2019 Conference

Marielle S. Gross, MD, MBE1, Carla Bossano, MD2, Nadine Rosenblum, RN, IBCLC3, and Lorraine Milio, MD, MPH2

Johns Hopkins Berman Institute of Bioethics, Johns Hopkins School of Medicine, and Johns Hopkins Hospital3; Baltimore, Maryland

Introduction: U.S. guidelines recommend breastfeeding women avoid marijuana given concerns about infant neurodevelopment. Unfortunately, this has resulted in many physicians and hospitals prohibiting women who use marijuana from breastfeeding despite inconclusive evidence of harm and well-known benefits of breastfeeding. Meanwhile, marijuana use is increasing among reproductive-aged women, and complex personal/socioeconomic factors affect feeding choices. We assess evidence and ethical justification for current practice.

Methods: We review: (1) Harm to infants from breastmilk marijuana exposure vs. avoiding breastfeeding, (2) Maternal health and psychosocial considerations, and (3) Current practices in light of principles of beneficence, justice, and autonomy.
Results: (1) First, delta-9-tetrahydrocannabinol (THC) is excreted in breastmilk and limited data (three studies with human subjects and three animal studies) suggest neurobehavioral changes among infants whose mothers use marijuana during breastfeeding, though effects of in utero vs. breastmilk exposure are difficult to distinguish and interpretation is limited by socioeconomic and other confounders. There are also concerns that marijuana use negatively impacts safe infant care and that the average concentration of THC in marijuana has increased in the years since the relevant studies were completed. Meanwhile, avoiding breastfeeding increases infants' risk of sudden infant death syndrome, sepsis, necrotizing enterocolitis, diabetes, asthma, and obesity. Available evidence is unclear regarding whether infants who were already exposed to THC in utero would be worse off with continued exposure through breastmilk vs. with increased risks associated with formula feeding. (2) Women who do not breastfeed have increased risk of cardiovascular disease, reproductive cancers, diabetes, depression and unintended pregnancy, all major sources of morbidity and mortality for U.S. women. Marijuana use is highest among underserved minority women who disproportionately suffer from the health consequences that breastfeeding may mitigate, and who are especially vulnerable to punitive damages (e.g., criminal charges related to drug use or Child Protective Services involvement). Maternal desire to bond with her infant through breastfeeding, cultural norms, values, and social pressures, and the financial burden of formula all may exacerbate the harm of telling women to avoid breastfeeding. (3) The national recommendation that women who breastfeed should avoid marijuana is interpreted by some physicians and hospitals as a policy that women who use marijuana should not breastfeed. This practice fails to account for the risks of avoiding breastfeeding for both infants and women, possibly exaggerating the strength of available evidence about harms from breastmilk THC exposure, and thus may not optimize health outcomes. In addition to potentially exacerbating existing health disparities, this practice may be unjust if women who screen positive for THC during pregnancy are told they are 'not allowed' to breastfeed regardless of whether they are actively using at time of delivery. Given the clinical equipoise regarding the best feeding method for infants whose mothers use marijuana, particularly if they were already exposed to THC in utero, and the large range in frequency/intensity of maternal marijuana use, an individualized, shared decision-making approach is appropriate. Furthermore, a woman's autonomy may 
be compromised if crucial postpartum lactation support is withheld while she is in the hospital postpartum or if she is concerned that breastfeeding against recommendations may jeopardize custody of her infant.

Discussion: Ultimately, failure to account for risks of avoiding breastfeeding for infants and women, with attention to epidemiology of marijuana use and breastfeeding-associated health effects, may result in policies which do not optimize health outcomes. Restrictive breastfeeding practices for women who use marijuana which do not utilize an individualized, shared decision-making approach are neither medically sound nor ethically justified, and may disproportionately undermine the health of underserved women and infants. Unbiased, culturally-informed and evidencebased counseling would promote open patient-provider communication may improve long-term health.

\section{NPA-2020-9}

Improving Staff Knowledge and Attitudes towards Providing Psychosocial Support to NICU Parents through an Online Education Course

Institutions: St. John's Regional Medical Center, Oxnard, CA, USA; University of Mississippi Medical Center, Jackson, MS, USA.

Authors: SL Hall MD; ME Famuyide MD; S Mosher RN, MHA; TA Moore RN, PhD; K Sorrells BSFCS; CA Milford EdS; J Craig PhD, MBA, OTRL, CNT; SN Saxton PsyD.

\section{Introduction}

Provider-parent communication is a critical determinant of how NICU parents cope with their situation and of how satisfied they are with their overall experience and with the care their infant received. NICU parents desire and benefit from psychosocial support from staff, and yet are not always satisfied with the communication and support they receive in the NICU. Many neonatologists and neonatal nurses do not feel they have adequate skills to communicate with distressed and anxious parents. Subsequently, this skill deficit increases staff's own stress and can contribute to on-the-job burnout. Additionally, as more NICUs are moving towards providing the model of family-integrated care, roles of NICU care providers are changing towards forming a more egalitarian partnership with parents and encouraging greater involvement of parents at their baby's bedside. This paradigm shift calls for new skill development among multidisciplinary staff.

\section{Objective}

This study sought to determine whether NICU staff would demonstrate improved knowledge and attitudes about their ability to provide psychosocial support to parents as a result of taking an online education course.

\section{Design}

This was a time series pre/posttest comparison of responses provided to a 33-item survey among NICU staff before and after taking an online education course on providing psychosocial support to parents. Content in the 7-hour course covered the categories as described in the "Interdisciplinary Recommendations for the Psychosocial Support of NICU Parents" (Hall and Hynan, J Perinatol, 2015).

\section{Setting}

Two NICUs participated in this project: St. John's Regional Medical Center (SJRMC), a 16-bed Level III community NICU with 250 admissions annually, and The University of Mississippi Medical Center (UMMC), a level IV academic NICU with 102 beds.

\section{Participants}

Staff at both NICUs, including physicians, nurses, occupational therapists, and social workers, were invited to take the online course and participate in the study.

\section{Methods}

Participants provided demographic information, then took a 33-item survey before (pretest) and after (posttest) taking the comprehensive course called "Caring for Babies and Their Families" using a Likert scale of 1-6 (1 = strongly disagree, $6=$ strongly agree). Pre- and posttest scores were analyzed using non-parametric paired t-tests.

\section{Results}

Of the 114 staff who registered for the course, $87.9 \%$ were nurses with a mean of 9.6 years of NICU service. All survey items showed posttest mean scores higher than pretest mean scores; in 30/33 (90.9\%) these differences were significant, $p<$ 0.05 . Night shift staff and staff with shorter periods of NICU service had lower pretest scores on several items; these differences were eliminated on the posttest. Educational needs for staff were identified on both the pretest and posttest.

\section{Discussion}

This education course was highly effective in improving staff knowledge and attitudes about how to support NICU parents, and in eliminating differences between day and night staff, and between those with shorter vs. longer periods of service in the NICU. Areas in need of further education were identified. Ninety percent of participants would recommend the course to their peers. Results are most applicable to nurses, who represented the majority of participants.

\section{NPA-2020-10}

Causal attributions of pregnancy loss amongst women who experienced fertility treatment

Alison R. Hartman, B.A., Victoria Grunberg, M.S., \& Pamela Geller, Ph.D.

Introduction: One in four women in U.S. will experience a pregnancy loss during their lifetime. Many women who experience a pregnancy loss attribute the loss to their own behaviors or character; that is, they blame themselves. Self-blame is also common among women who experience infertility, and is associated with adverse psychosocial consequences including an increased suicide risk and decreased relationship satisfaction. It remains unclear as to whether women who have experienced pregnancy loss after conceiving with fertility treatment attribute the cause of their loss differently than women who have experienced a pregnancy loss without undergoing fertility treatment. The current study was conducted to examine whether women who underwent fertility treatment tend to blame themselves for their loss more often than women who did not.

Methods: The present online survey study includes women from diverse backgrounds ( $\mathrm{N}=825$; Age: 18 - 66 years, $\mathrm{M}=$ $31.87, \mathrm{SD}=8.69$ ) who have experienced a pregnancy loss at some point in their lifetime. By utilizing social media platforms, a large proportion of minority women were included in the sample ( $N=391 ; 47.40 \%)$. Participants reported whether fertility treatment was used to achieve the index pregnancy, and self-reported causal attributions of their most recent loss using the Pregnancy Loss Attributional Questionnaire. Examples from this questionnaire include, If I were a different age, this loss might not have happened; Others deserve to be a parent more than I did, which helps to explain why I had the loss; and If I rested more, the loss might not have happened.

Results: Descriptive statistics indicate that, on average, women's most recent pregnancy loss occurred $4.21 \pm 3.55$ years prior to the time of survey completion ( $\mathrm{N}$ 
$=825)$. Further, $5.78 \%$ of participants $(n=$ 48 ) reported that they used fertility treatment to achieve the pregnancy that was lost. Independent t-tests were conducted to determine which causal attributions (e.g., external attribution, internal characterological attribution, or internal behavioral attribution) were more prevalent among women who experienced a pregnancy loss following fertility treatment compared to women who experienced a loss without fertility treatment. Results indicated that women who underwent fertility treatment were more likely to attribute their loss to their age, $\mathrm{t}(668)=-2.49, \mathrm{p}=.013$, and lack of rest, $\mathrm{t}(668)=-2.33, p=.020$. Further, women who underwent fertility treatment were more likely to report that the loss was related to punishment for "the person I am," $\mathrm{t}(668)=-1.92, p=.056$, and that "others deserve to be a parent more than I did," $\mathrm{t}(668)=-2.79, \mathrm{p}=.005$.

Discussion: Findings indicated that women who underwent fertility treatment were more likely to blame their age and lack of rest for the loss. Notably, these women were also more likely to endorse that they were less deserving of parenthood and that the loss was a form of punishment. Women who undergo fertility treatment and experience pregnancy loss may be at increased risk for negative psychosocial sequelae due to elevated feelings of self-blame. It is important that healthcare providers, clinicians, and researchers be aware of the role of self-blame within these reproductive life events to facilitate psychoeducation and open communication with patients.

\section{NPA2020-11}

\section{Innovative Models of Care:}

\section{Neonatal Social Work Care Coordina- tion in the NICU and NICU Follow-up Programs}

Author: Ryan Nicoll, MSW, LISW-S; ryan. nicoll@nationwidechildrens.org

Nationwide Children's Hospital, 700 Children's Drive, Columbus, Ohio 43205

Background: According to the Council on Children with Disabilities and Medical Home Implementation Project Advisory Committee, "Care Coordination is an essential element of a transformed American health care delivery system that emphasizes optimal quality and cost outcomes, addresses family-centered care, and calls for partnerships across various settings and communities." The NICU Follow-up Program at Nationwide Children's Hospital monitors the developmental progress of eligible NICU graduates until the age of 3 years, but retention rates have been variable. The need for education and guidance to NICU families regarding recommendations for their child's follow-up and a process to identify and problem-solve barriers to care was much needed. This led to the development of Neonatal Social Work Care Coordination Services (NEOSWCCS). This specialized social work program specifically addresses the transition from hospital to home and provides partnership with families to help them better understand the goals of developmental surveillance and intervention as well as problem solve practical barriers to care which may interfere with program retention.

Content/Action: The poster will showcase this specialized program, Neonatal Social Work Care Coordination Services (NEOSWCCS), which was initiated in October of 2016. Patients discharged from the NICU are eligible for NEOSWCCS if they meet specific criteria potentially associated with non-adherence (e.g. parents with cognitive limitations, mental health issues, language barriers) or if the child's healthcare needs are especially complex. Once a patient is identified as eligible for NEOSWCCS, attempts are made by the Neonatal Social Work Care Coordinator (Neo SWCC) to meet families referred to the NICU Followup Program prior to their discharge from the NICU. The Neo SWCC provides parent education about the clinic their child will be attending and the importance of developmental monitoring and intervention. Barriers to follow-up care are also explored during this initial face-to-face intake and the Neo SWCC then links families with available resources to mitigate these barriers. In addition, the Neo SWCC completes a phone call approximately one week after discharge to asses for post-discharge needs and during the week prior to the initial developmental evaluation (typically at 3-4 months corrected age) to provide information regarding what to expect for the evaluation and explore barriers to care. The Neo SWCC has also led an initiative developing systems to follow up on noncompliance in the clinics which includes a triage process for high risk patients. This has been a multidisciplinary effort rolled into standard operating procedures for the clinics.

Lessons Learned: Developing clear criteria for patient eligibility and having pre-existing clinical relationships with the multidisciplinary team in the NICU was essential. Both helped to identify patients, facilitate communication with the families, and for identification of barriers to care. Challenges during the implementation of this program include slower or missed identification of eligible families for NEO-
SWCCS during planned and unplanned absences of the Neo SWCC as well as an insufficient tracking system to evaluate circumstances affecting data.

Implications of Practice: The implementation of NEOSWCCS allows for targeted interventions specific to helping families transition from their NICU care to outpatient follow-up thus increasing the retention rates and developmental follow-up. The NICU Follow-up Program at Nationwide Children's Hospital averages 5,000 completed visits each year. Average completion rate of the $\mathrm{D} 1$ developmental evaluation (3-4 months corrected age) in the NICU Follow-up Program in 2016 was $52 \%$. In 2018 , the rate increased to $89 \%$ (for completion of initial developmental evaluation) for patients eligible for NEOSWCCS. Results will be illustrated through tables and will include data from 2019.

\section{NPA2020-12}

\section{Innovative Models of Care:}

\section{Neonatal Social Work Care Coordina- tion in the NICU and NICU Follow-up Programs}

Author: Ryan Nicoll, MSW, LISW-S; ryan. nicoll@nationwidechildrens.org

Nationwide Children's Hospital, 700 Children's Drive, Columbus, Ohio 43205

Background: According to the Council on Children with Disabilities and Medical Home Implementation Project Advisory Committee, "Care Coordination is an essential element of a transformed American health care delivery system that emphasizes optimal quality and cost outcomes, addresses family-centered care, and calls for partnerships across various settings and communities." The NICU Follow-up Program at Nationwide Children's Hospital monitors the developmental progress of eligible NICU graduates until the age of 3 years, but retention rates have been variable. The need for education and guidance to NICU families regarding recommendations for their child's follow-up and a process to identify and problem-solve barriers to care was much needed. This led to the development of Neonatal Social Work Care Coordination Services (NEOSWCCS). This specialized social work program specifically addresses the transition from hospital to home and provides partnership with families to help them better understand the goals of developmental surveillance and intervention as well as problem solve practical barriers to care which may interfere with program retention.

Content/Action: The poster will showcase 
this specialized program, Neonatal Social Work Care Coordination Services (NEOSWCCS), which was initiated in October of 2016. Patients discharged from the NICU are eligible for NEOSWCCS if they meet specific criteria potentially associated with non-adherence (e.g. parents with cognitive limitations, mental health issues, language barriers) or if the child's healthcare needs are especially complex. Once a patient is identified as eligible for NEOSWCCS, attempts are made by the Neonatal Social Work Care Coordinator (Neo SWCC) to meet families referred to the NICU Followup Program prior to their discharge from the NICU. The Neo SWCC provides parent education about the clinic their child will be attending and the importance of developmental monitoring and intervention. Barriers to follow-up care are also explored during this initial face-to-face intake and the Neo SWCC then links families with available resources to mitigate these barriers. In addition, the Neo SWCC completes a phone call approximately one week after discharge to asses for post-discharge needs and during the week prior to the initial developmental evaluation (typically at 3-4 months corrected age) to provide information regarding what to expect for the evaluation and explore barriers to care. The Neo SWCC has also led an initiative developing systems to follow up on noncompliance in the clinics which includes a triage process for high risk patients. This has been a multidisciplinary effort rolled into standard operating procedures for the clinics.

Lessons Learned: Developing clear criteria for patient eligibility and having pre-existing clinical relationships with the multidisciplinary team in the NICU was essential. Both helped to identify patients, facilitate communication with the families, and for identification of barriers to care. Challenges during the implementation of this program include slower or missed identification of eligible families for NEOSWCCS during planned and unplanned absences of the Neo SWCC as well as an insufficient tracking system to evaluate circumstances affecting data.

Implications of Practice: The implementation of NEOSWCCS allows for targeted interventions specific to helping families transition from their NICU care to outpatient follow-up thus increasing the retention rates and developmental follow-up. The NICU Follow-up Program at Nationwide Children's Hospital averages 5,000 completed visits each year. Average completion rate of the D1 developmental evaluation (3-4 months corrected age) in the NICU Follow-up Program in 2016 was $52 \%$. In 2018, the rate increased to $89 \%$ (for completion of initial developmental evaluation) for patients eligible for NEO-
SWCCS. Results will be illustrated through tables and will include data from 2019.

\section{NPA2020-12}

Family Infant Neurodevelopmental Education (FINE)

Poster proposal for the National Perinatal Association Meeting

Author: Debra Paul, BS, OTR/L (parent) and Joy V. Browne, Ph.D., PCNS, IMH-E (IV),

Background: Developmental care is a globally accepted and evidence based approach to optimizing outcomes for babies and their families. Through the work of Dr. Heidelise Als, Beverly Johnson and others, and now with recommended standards and competencies in Europe, Canada and the United States, developmental, family centered care is becoming the expected norm. The gold standard for education and implementation of this approach is the Newborn Individualized Developmental Care and Assessment Program (NIDCAP; www.nidcap.org) which has 22 training centers worldwide. However, training in the NIDCAP program is complex and has not been well accepted as a model in the US. In the past decade a foundational program, referred to as Family Infant Neurodevelopmental Education (FINE) program was developed in Europe to meet the needs of NICU professionals who wish to have more empirically supported strategies for implementing basic practice in neurodevelopmental care. There are two levels of the FINE program, a two day foundational education program for all NICU professionals (FINE 1) and a 12 week individualized program for those who wish to have a more in depth mentored experience incorporated into their practice (FINE 2). Both are intended to be foundational for those who wish to become NIDCAP Professionals.

Content/Action: In 2019 the two day FINE 1 program was implemented in US locations with over 320 interdisciplinary professionals. At the conclusion of FINE 1 training, attendees identified a variety of areas where they want to implement infant and family supportive strategies into their NICU caregiving. Themes included: enhanced integration of families in infant care, more consistent kangaroo mother care, pain prevention and alleviation, avoidance of sleep disruption, better positioning and alignment for babies, and demand feeding practices. An overview of the components of FINE training that are most salient for NICU professionals as well as specific data regarding how attendees plan to utilize the information from the FINE 1 program will be provided. One year fol- low up data are currently being obtained to determine long term follow through on how attendees have implemented evidence based family centered and developmental care practices.

Lessons learned: The FINE program appears to be well accepted and has implications for evidence based developmental care. It has been developed to be consistent with the Gravens Standards and Competencies for Infant and Family Centered Developmental Care (see abstract by Browne and Jaeger) and the European Foundation for the Care of Newborn Infants (EFCNI) standards for newborn health in Europe.

Implications for practice: Neurodevelopmental care practices are evidence based with standards for implementation in all NICUs. FINE 1 training provides foundational training that is consistent with best practice and provides rationale for optimizing infant and family support during hospitalization. With data now being accumulated, we will have a better understanding of what practices are consistently implemented and utilized as a result of attending the FINE 1 training.

\section{NPA2020-13}

The impact of sociodemographic characteristics on postpartum depression in Hispanic women

Authors: Sneha Rajendran, BS,BA, Mary S. Dietrich, PhD, MS, Melanie Lutenbacher, PhD, MSN, RN, FAAN

INTRODUCTION: Hispanic people living in the United States "bear a disproportionate burden of disease, injury, death, and disability" when compared to non-Hispanic white people(1). Postpartum depression falls into this category. Despite similar rates of postpartum depression in women of differing ethnicities, among low-income women, the odds of starting and continuing treatment for postpartum depression following delivery are significantly lower for Hispanic women compared to white women(2). Barriers to care has been hypothesized as a potential explanation, but has not been supported(3). Other possible factors that may contribute to the healthcare disparity Hispanic women with postpartum depression face must be examined. Evidence suggests that various sociodemographic characteristics and maternal factors such as age(4), breastfeeding duration(5), and intimate partner violence(6) may be associated with postpartum depression. This study further examines these and other maternal factors and their potential relationship with reliable change in the levels of depressive symp- 
toms from late pregnancy to two months and six months postpartum in a sample of Hispanic women living in Davidson County, TN.

METHODS: Data for this secondary analysis were collected in an RCT conducted from July 2014 to September 2016 which assessed the efficacy of the Maternal Infant Health Outreach Worker (MIHOW) program (www.mihow.org), a peer mentoring home visitation program, in a sample of 188 Hispanic women(7). A prospective, longitudinal experimental design with two study groups: comparison (printed educational material) and intervention (MIHOW home visits plus printed educational material) was used. Eligibility criteria included: age $\geq 18$, self-identification as Hispanic, confirmation of pregnancy $\leq 26$ weeks gestation, and residence within 30 miles of study offices. Data was collected at five time points (prenatal through six months postpartum) using validated measures and questions from national surveys. The study was approved by the Vanderbilt University Institutional Review Board. The sample for the secondary analysis included the 178 participants who completed the parent study and their de-identified data related to: levels of depressive symptoms, acculturation, health literacy, parenting stress, and education, breastfeeding intent, duration, and self-efficacy, time living in the US, maternal age, presence of a medical provider, health insurance, and presence of infant NICU stay. Multivariate logistic regression was used to analyze the significance of each of these demographic variables in explaining variance in reliable change in level of depressive symptoms. The following three variables were used as co-variates to control for changes in the outcome variable: 1) gestational age at study enrollment, 2) level of depressive symptoms at baseline, and 3) parent study group assignment.

RESULTS: The average maternal age at enrollment was 29.6 years $(S D=6.5)$. The median gestational age was 17.5 weeks. The median time lived in the USA was 9 years (IQR=3-13). Mexico had the largest representation of home country $(66.9 \%)$, followed by Honduras (15.7\%) and El Salvador $(9.6 \%) .19 .3 \%$ of the subjects had graduated high school or completed a GED. $68.5 \%$ of the subjects earned less than $\$ 10,000$ yearly in family income, and $28.1 \%$ earned between $\$ 10,0001$ $\$ 15,000$. Of the factors examined, the presence of health care coverage at two months postpartum was associated with a statistically significant decrease in level of depressive symptoms ( $p=0.017,95 \%$ Cl 1.279 - 12.763) and a higher parental stress score at six months postpartum was associated with a statistically significant increase in level of depressive symptoms ( $p$
$=0.02,95 \% \mathrm{Cl} 0.842-0.986)$.

DISCUSSION: The findings have clinical and research implications. Helping patients access available health care coverage and resources that may help lower their parenting stress are important factors to consider when caring for Hispanic women, particularly those with postpartum depression. Future research related to postpartum depression should include these variables and potential evaluation of interventions that may impact change. Further research into this healthcare disparity will increase our understanding of characteristics and maternal factors that may contribute to variability of depressive symptoms in Hispanic women and serve as the underpinnings for targeted culturally competent interventions and policies for a growing minority in the United States.

\section{REFERENCES}

(1) CDC: Health Disparities Experienced by Hispanics --- United States

(2) Kozhimannil, K. B., Trinacty, C. M., Busch, A. B., Huskamp, H. A., \& Adams, A. S. (2011). Racial and ethnic disparities in postpartum depression care among low-income women. Psychiatric Services, 62(6), 619-625.

(3) Salameh, T. N., Hall, L. A., Crawford, T. N., Staten, R. R., \& Hall, M. T. (2019). Racial/ethnic differences in mental health treatment among a national sample of pregnant women with mental health and/ or substance use disorders in the United States. Journal of psychosomatic research, 121, 74-80.

(4) Robbins, C., Boulet, S. L., Morgan, I., D’Angelo, D. V., Zapata, L. B., Morrow, B., ... \& Kroelinger, C. D. (2018). Disparities in preconception health indicators-Behavioral risk factor surveillance system, 20132015, and pregnancy risk assessment monitoring system, 2013-2014. MMWR Surveillance Summaries, 67(1), 1.

(5) Lara-Cinisomo, S., McKenney, K., Di Florio, A., \& Meltzer-Brody, S. (2017). Associations between postpartum depression, breastfeeding, and oxytocin levels in Latina mothers. Breastfeeding Medicine, 12(7), 436-442.

(6) Ogbo, F. A., Kingsley Ezeh, O., Dhami, M. V., Naz, S., Khanlari, S., McKenzie, A., ... \& Eastwood, J. (2019). Perinatal distress and depression in culturally and linguistically diverse (CALD) Australian women: the role of psychosocial and obstetric factors. International journal of environmental research and public health, 16(16), 2945. (7 ) Lutenbacher, M., Elkins, T., Dietrich, M.S., Riggs, A. (2018). The Efficacy of using peer mentors to improve maternal and infant health outcomes in Hispanic families: Findings from a Randomized Clinical Trial. Maternal and Child Health Journal, 22 (supplement 1), 92-104.
OPIOIDS and NAS

When reporting on mothers, babies, When reporting on

LANGUAGE MATTERS

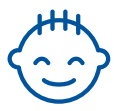

I am not an addict

was exposed to substances in utero. I am not addicted. Addiction is a set of behaviors associated with having a Substance Use Disorder (SUD).

I was exposed to opioids. While I was in the womb my mother and shared a blood supply. I was exposed to the medications and substances she used. I may have become physiologically dependent on some of those substances.

NAS is a temporary and treatable condition.

There are evidence-based pharmacologica and non-pharmacological treatments for Neonatal Abstinence Syndrome.

\section{My mother may have a SUD.}

She might be receiving Medication-Assisted Treatment (MAT). My NAS may be a side effect of her appropriate medical care. It is not evidence of abuse or mistreatment.

\section{My potential is limitless}

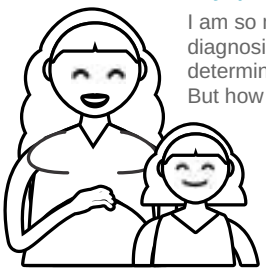

PO NAS ingosis. My drug exposure will not my long-term outcomes. ill. When you invest in my family's health and wellbeing by supporting Medicaid and Early Chilcid and Early Childhood Education you can expect that I will do as well as any of my peers
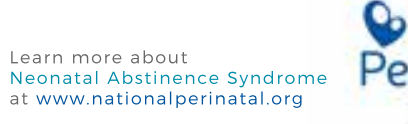
National erinatal

NPA2020-14

NPA ABTRACT SUBMISSION (for poster presentation)

TITLE OF ABSTRACT Family Celebrations: A NICU Perspective Navy Spiecker, BA, Pamela A. Geller, PhD, Chavis A. Patterson, PhD

Background:For many parents, celebrations can be a joyful time; however, for those with an infant in the NICU, holidays can cause conflicting emotions. Parents face difficulties integrating celebration with the anxiety they may be simultaneously experiencing. Additionally, parents may feel isolated as they manage their infant's illness or bereavement while other families participate in celebratory activities. This project seeks to offer a greater understanding of the complex emotions families face with regard to child illness during holidays. The goal is to summarize 
the literature and offer recommendations to NICU providers on how to best assist families around celebratory events.

Content/Action: Existent literature on family experiences in the NICU during holidays was examined, including: review of academic articles, qualitative examination of personal stories from families, and input from NICU providers.

Lessons Learned: Recommendations are made towards financial/transportation support available to families, the utilization of parent support and activity groups, integrating volunteer assistance from previous graduate families of the NICU, and utilizing a family-centered approach to care with regard to celebrations and holidays. Additionally, emphasis is placed on the provider's knowledge of outside resources/nonprofits dedicated to supporting families in the NICU.

Implications for Practice: A thorough understanding of the family's experience in the NICU during celebrations will help providers address challenges with effective evidence-based care. Provision of open dialogue, celebratory programs for parents within the NICU, and knowledge of outside resources can improve coping among parents. Current literature and resources in this area are limited.

Providers should consider the role of outside factors that further complicate the NICU experience, such as time divided between home and the bedside.

\section{NPA2020-15}

\section{Innovative Models of Care}

Petora Spratt, P.T., D.P.T., IMH-E (III)Emily McNeil, L.C.S.W, IMH-E (IV), Debra Paul, OTR (parent), and Joy Browne, Ph.D., PCNS, IMH-E (IV).

Background: Infants and their families who transition from NICU to their communities are typically followed by early intervention and/or public health nurses. Medical complications, invasive procedures and many unknowns during hospitalization for both infants and their families result in physical, mental and behavioral health issues that require appropriately informed mental health supports. Currently there is little mental health information and/or approaches in basic educational programs for providers that address the developmental and mental health issues of newborns and young infants. The BABIES and PreSTEPS model has been developed to provide providers in the community with appropriate education to address mental, physical and developmental health issues of this vulnerable population and their fam- ilies.

Content: Data will be provided from surveys of providers in four states (AK, CO, IN and $A Z$ ) indicating a lack of specific training for physical, developmental and mental health assessment and intervention for fragile newborns and their families. Description of the BABIES (Biophysiologic, Arousal and Sleep, Body Movement, Interaction with others, Eating and Soothing) and PreSTEPS (Predictability and continuity, Sleep and arousal supports Timing and pacing, Environmental modification and Soothing strategies) model will be presented to include assessment and intervention guidance for supporting fragile newborns and their parents in the fourth trimester. Infant Mental health Diversity Tenets and Reflective Practice best practices are used in the year long learning collaborative. Descriptions of state wide provider practice outcomes as a result of engaging in the educational program will be provided.

Lessons learned: A mental health informed practice including reflective opportunities are essential to best support fragile newborns and young infants and their families in the fourth trimester. Although essential to the recovery of infants and parents after hospitalization, providers do not have the educational background to incorporate mental health approaches into their currently utilized intervention strategies. Parents are the best supporters of regulation in the fourth trimester, an essential developmental task of newborns. Support for both providers and families during this vulnerable time is essential.

\section{Implications for practice:}

\section{NPA2020-16}

\section{NPA Innovative Model of Care Proposal}

Title: Baby Attachment and Comfort Interventions (BACl): A multidisciplinary intervention to support parents and neonates in a cardiac neonatal intensive care unit in the first weeks after birth

Authors: Rochelle Steinwurtzel, Katharine Press Callahan, Elvira Parravicini

Background: Parents with babies in the Neonatal Intensive Care Unit (NICU) due to congenital heart disease (CHD) are at high risk for stress in the context of longlasting emotional, familial and financial costs associated with diagnosis, hospitalizations, and ongoing treatment. Babies hospitalized in the NICU for CHD often experience multiple traumas related to physically stressful medical procedures while experiencing a loss of other developmentally appropriate sensory inputs.
Simultaneously, they experience the additional stressor of separation from their parents whose role is to facilitate a sense of security through a constant, loving and responsive relationship.

It is essential for parents to buffer infants' stress levels. If parents are overwhelmed by their own stress levels associated with the NICU experience, their capacity to effectively regulate the baby's stress becomes compromised. Trauma-informed care in the NICU empowers staff to support parents and in turn neonates. Similarly, palliative care principles focus on improving quality of life and reduce suffering while enhancing families' decision-making capabilities through early integration of interdisciplinary interventions.

Content/Actions: The Baby Attachment Comfort Interventions (BACl) is an innovative method of early palliative care developed and validated by the Neonatal Comfort Care Program at NewYork Presbyterian, Columbia University Irving Medical Center in an effort to support parents and enhance comfort of all hospitalized neonates, regardless of prognosis (Callahan, K., Steinwurtzel, R., Brumarie, L., Schechter, S., \& Parravicini, E. Early palliative care reduces stress in parents of neonates with congenital heart disease: validation of the "Baby, Attachments, Comfort Interventions. J Perinatology. 2019; 39(12):1640-1647). BACI utilizes palliative care and trauma-informed care concepts with a focus on supporting parents so they can more effectively co-regulate their hospitalized babies. $\mathrm{BACl}$ focuses on four domains: bonding, feeding, memories, and emotional, psychological, and spiritual support. The intervention is provided by the interdisciplinary $\mathrm{BACl}$ team, which includes the Neonatal Comfort Care Program core team (a neonatologist/medical director, nurse, and social worker) and other NICU professionals including a psychologist, speech pathologist, Child Life specialist, and chaplain. Overall, $\mathrm{BACl}$ team members meet with parents an average of 4 times per week and offer a variety of services that are tailored to the individual family and the neonate's medical condition. Services include opportunities for skin-to-skin, developmentally-appropriate touch and positioning, non-nutritive suck or colostrum care, memory-making, and psychological and spiritual support. Additionally, BACl helps support bedside staff and foster opportunities for parental involvement in pleasurable dyadic experiences between parent and baby.

Lessons Learned: Based on previously published findings (Callahan et al., 2019), the $\mathrm{BACl}$ program significantly reduces stress in parents of infants with CHD. The $\mathrm{BACl}$ program requires the focused attention and availability of the $\mathrm{BACl}$ team, in 
addition to their regular job roles.

Implications: This program requires dedicated time and resources to provide the consistent, multidisciplinary care parents need to feel psychologically safe in the cardiac NICU. Future research could assess whether effects on parental stress persist long-term or how this program impacts the stress of staff.

\section{NPA2020-17}

\section{Redefining the Postpartum Care Rota-} tion for OB/Gyn Interns

Authors: Julia Switzer, MD; Aref Senno, MD; Kavisha Khanuja, MD; Abigail Wolf, MD

Background: Increased recognition of the importance of the 'fourth trimester' and the pressing need to reduce maternal morbidity and mortality, has led many professional organizations, including ACOG, to encourage a renewed focus on postpartum care. Changing the culture of practice requires changing the way we teach our trainees. Resident training in OB/Gyn is rigorous and historically has not allowed for focused study of the postpartum period. Postpartum rounding is typically done early, quickly, and as an afterthought to other responsibilities such as managing patients on Labor and Delivery and in Triage. The ACGME Milestones Project helps to define the developmental steps necessary for a resident to move towards independent practice. Advanced milestones for The Care of the Postpartum Patient include ability to effectively counsel patients on antenatal, intrapartum and postpartum complications, collaboration with other members of the health care team in postpartum care and application of innovative approaches to the management of patients in the postpartum period. There is currently no literature regarding how to teach OB/Gyn residents about comprehensive postpartum care.

Action: In order to emphasize the importance of the fourth trimester, we created a postpartum care rotation to allow time for the resident to provide culturally sensitive and individualized care, be directly observed and receive feedback in the postpartum care environment, and to learn about the complications of the postpartum period. Under the guidance of their attending, the resident independently manages the care of the postpartum service. Care coordination is a large part of the rotation. The resident works with hospital social workers, case management, lactation consultants, medical consultants and outpatient practice members to individualize the outpatient follow up needs of all patients while learning to manage an inpatient service. During this block the resident also staffs a dedicated outpatient postpartum clinic two afternoons per week. Assigned learning tasks of this rotation include: completion of a breastfeeding training course; direct observation of the informed consent process, implicit bias training with reflection and discussion of perinatal mood disorders.

Lessons Learned: Feedback regarding this rotation was collected through resident interviews. Recognized benefits of the rotation include: perception of appropriate time for counseling specifically around contraceptive choices in medically complex patients, understanding of lactation and feeding concerns, decreased stress regarding the time spent on the postpartum unit (and therefore away from Labor and Delivery or other responsibilities) while counseling patients, increased utilization of video interpreter services, and generation of ideas for quality improvement projects. Residents have the opportunity for continuity in that they can schedule and see patients in the outpatient setting whom they have cared for while inpatient. One-on-one rounding with the attending provides more opportunity for direct observation of patient care, patient handoffs, discharge planning and patient counseling. Patients are also invited to provide feedback on the resident's professionalism, communication skills and medical care. Concerns about the rotation included an increased burden of administrative paperwork on the resident and a sense of highly repetitive work. Faculty development is needed in order to change the approach to the postpartum rounding and allow for direct teaching with the resi- dent.

Implications for Practice: By creating a dedicated postpartum rotation with specific learning goals and objectives, we are demonstrating to our trainees that this aspect of care is critically important to development as an Ob/Gyn physician. Participation in a focused postpartum rotation may therefore improve attention to this aspect of care once the resident enters independent practice. In addition, the postpartum unit is an optimal environment to promote interprofessional education and teamwork. Direct observation in this environment allows for timely feedback on performance which aligns with the ACGME milestones for OB/Gyn Residency Training.

\section{NPA2020-18}

Innovative Models of Care: Essential Knowledge and Competencies for Psychologists Working in Neonatal Intensive Care Units (POSTER)

Authors: Willis, T., Saxton, S., Dempsey, A., Baughcum, A., Chavis, L., Hoffman, C., Fulco, C., Milford, C., \& Stenberg, Z

Background: The role of the neonatal psychologist is multifaceted, with psychologists embedded in inpatient NICUs, outpatient NICU follow-up developmental clinics, and fetal care centers. Consistent with efforts of other sub-specializations to delineate training and competency guidelines to prepare psychologists in subspecialty fields (e.g., Jerson, Cardona, Lewallen, Coleman, \& Goyette-Ewing, 2015; McDaniel et al., 2014; Palermo et al., 2014), the proposed poster will present an aspirational model that begins to define competency in the sub-specialization of neonatal psychology. Our general framework was adapted from a paper on training and competency standards for psychologists in primary care (McDaniel et al., 2014), which was based on competency models in psychology that focus on achievement of measurable, behavioral learning objectives rather than a focus on curriculum (Kaslow, 2004). The model includes six clusters: Science, Systems, Professional-

NEONATOLOGY TODAY is interested in publishing manuscripts from Neonatologists, Fellows, NNPs and those involved in caring for neonates on case studies, research results, hospital news, meeting announcements, and other pertinent topics. Please submit your manuscript to: LomaLindaPublishingCompany@gmail.com 
ism, Relationships, Application, and Education. Each of these clusters is subdivided into associated competency groups, and each of which has its own table with specific knowledge/skills.

Content/Action: To identify the key knowledge and abilities to be included within each competency table, the workgroup evaluated literature of behavioral health issues that present in NICUs and consulted a number of different groups that included NICU psychologists, physicians, clinicians, therapists, and parents. Over a 2-year period (2017-2019), the workgroup generated a list of key knowledge and abilities for each competency group. Once all tables were populated, each workgroup member reviewed all material contained across the competency tables and identified areas of overlap within and across tables, added additional items they felt were omitted, and indicated the six to ten over-arching themes that summarized the items within each competency group.

Lessons Learned: It is important to note that the identified areas of knowledge and abilities are provided as a general reference and are not intended to be prescriptive. Psychologists pursuing this area of subspecialty are not expected to have expertise in all of these areas. The utility of each competency and specific knowledge area will vary depending on the psychologist's role, setting, time dedicated to NICU work, and/or service level of the NICU.

Implications for Practice: Given the array of expectations for neonatal psychologists, specialized training that goes beyond the basic competencies of a psychologist in general practice and includes a wide range of learning across multiple domains is needed. For both trainees and practicing psychologists who seek to work as a neonatal psychologist, we strongly recommend seeking education and training in (1) infant mental health, focusing on the dyadic relationship; (2) identification and treatment of perinatal mood and anxiety disorders and trauma; (3) family systems practice and impact of pediatric medical condition on coping/adjustment, and (4) provision of integrated mental health services in a medical setting. Additionally, the neonatal psychologist's role may vary greatly across NICUs; the ability to conduct a needs assessment and develop and evaluate programs is critical, particularly when establishing new psychological services. Achieving competency will enable the novice neonatal psychologist a more successful transition into a highly complex, fast-paced, often changing medical environment, and ultimately, provide the best care for the infants and their families.

\section{NPA2020-19}

Revisiting the Postpartum Home Visit: A Call to Action

\section{Author: Yeman, Jodi}

Background: Postpartum health care has been reduced to a 48-96 hour hospital stay depending on the type of delivery, followed by a 6 week postpartum clinic visit that marks the end of the postpartum period by all conventional standards. The United States maternal mortality rate continues to climb with a $26.6 \%$ increase from 2000 to 2014 . Approximately $15-20 \%$ of postpartum women will develop postpartum depression within the first year of after delivery which has generated much discussion surrounding the most effective way to identify those at risk and provide adequate support and management. In light of these statistics the conversation surrounding how to best meet the postpartum needs of women and newborns has been renewed.

In 2018 the American College of Obstetricians and Gynecologists (ACOG) proposed redesigning postpartum care with the goal of providing a more holistic approach to what is known as the $4^{\text {th }}$ trimester, addressing areas such as mood and emotional well-being, maternal infection, infant care and feeding along with addressing sleep and fatigue issues to name a few.

A successful postpartum home visit program addressing the $4^{\text {th }}$ trimester already exists that encompasses many of ACOG's goals. The Duke Family Connects model has been studied in two randomized controlled trials demonstrating improved mother mental health, reduced emergency care for participating infants of $59 \%$, enhanced home environments and greater community connections to programs like Nurse-Family Partnership for continued long-term continuity of care. The Family Connects program studied demonstrated that for each program $\$ 1$ spent, a savings of $\$ 3.04$ in emergency care costs was produced.

Content/Action: Postpartum home visits should be incorporated as the standard of care for pregnant women and considered part of the multidisciplinary team that sup- ports and cares for new families during this critical life transition using the Duke Family Connects as a model. Current evidence supports the benefits of providing home nurse visits in reducing readmission rates for both newborns and mothers as well as promoting family bonding.

Lessons Learned: Successful postpartum home visit programs should begin before the family is discharged home. The home visit nurse needs an opportunity to establish rapport with the family and time to assess and evaluate their unique needs prior to delivery. Many women find it challenging and burdensome to make multiple doctors visits once the baby arrives. Home visits are patient centered and scheduled around convenience for the family. A postpartum home visit program can facilitate individualized transition of care plans to community resources for those families that need continued care beyond the $4^{\text {th }}$ trimester.

Implications for Practice: With the Postpartum home visit model as the standard of care, women will have access to quality care that is timely and holistic. Postpartum home visit studies to date reflect improved outcomes for both mom and newborn as well as reduction in cost related to decreased readmissions. Successful programs already exist and include interactions with and assessment of the family prior to delivery. Ultimately, if implemented as part of the standard of care for childbearing families, postpartum home visits could bridge the gap in care during the $4^{\text {th }}$ trimester and reduce maternal and infant morbidity and mortality in the United States.

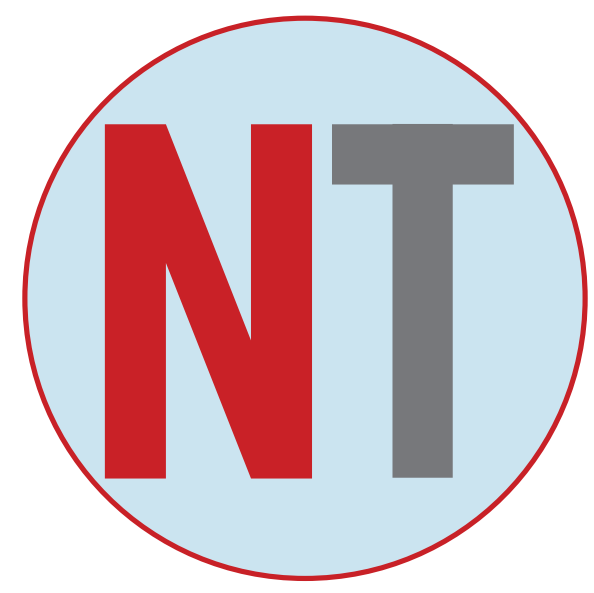


Disclosure: The National Perinatal Association www.nationalperinatal.org is a 501c3 organization that provides education and advocacy around issues affecting the health of mothers, babies, and families.

NT
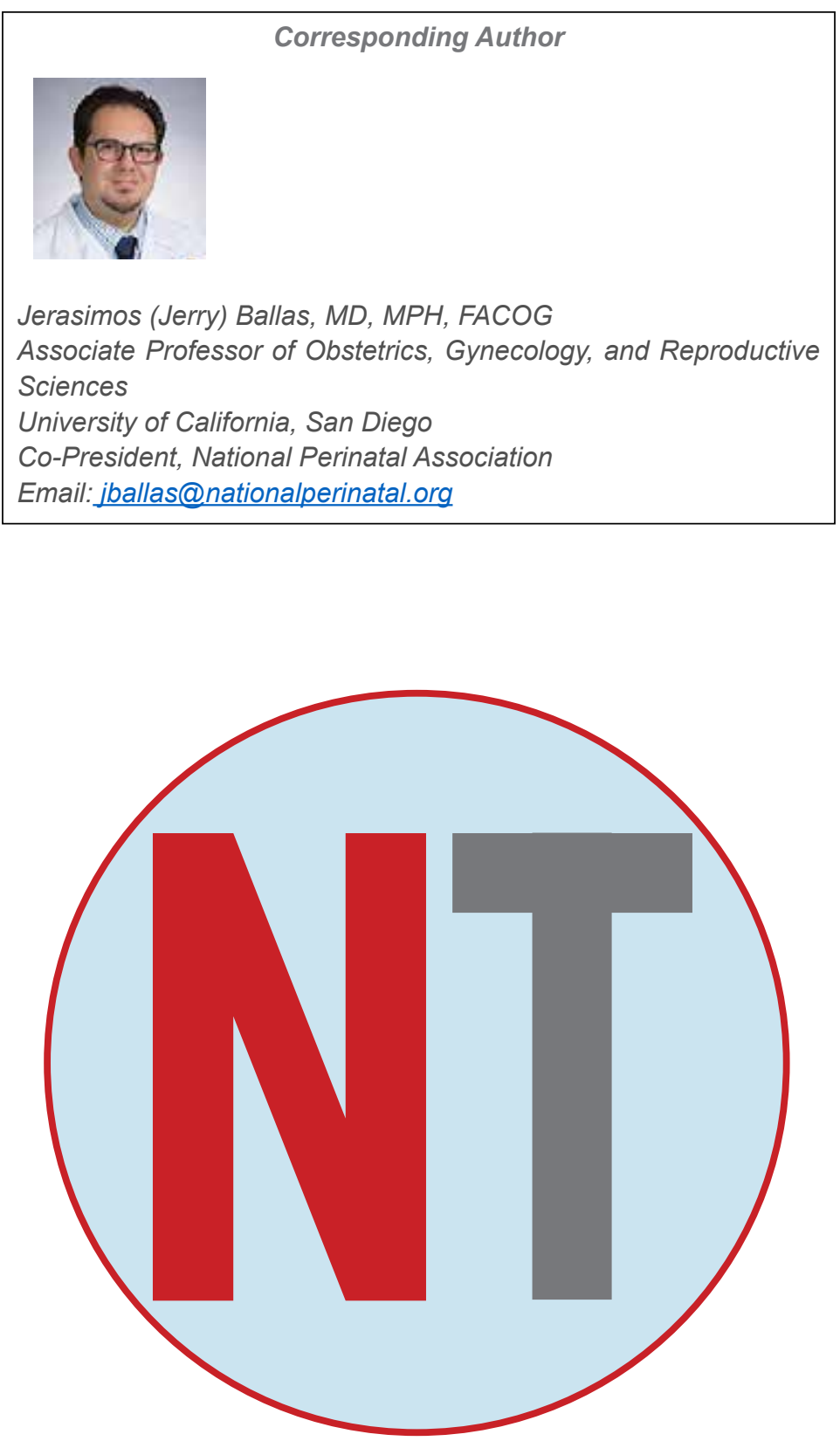

New subscribers are always welcome! NEONATOLOGY TQDAY

To sign up for free monthly subscription, just click on this box to go directly to our subscription page

\section{Readers can also follow NEONATOLOGY TO via our Twitter Feed @NEOTODAY}

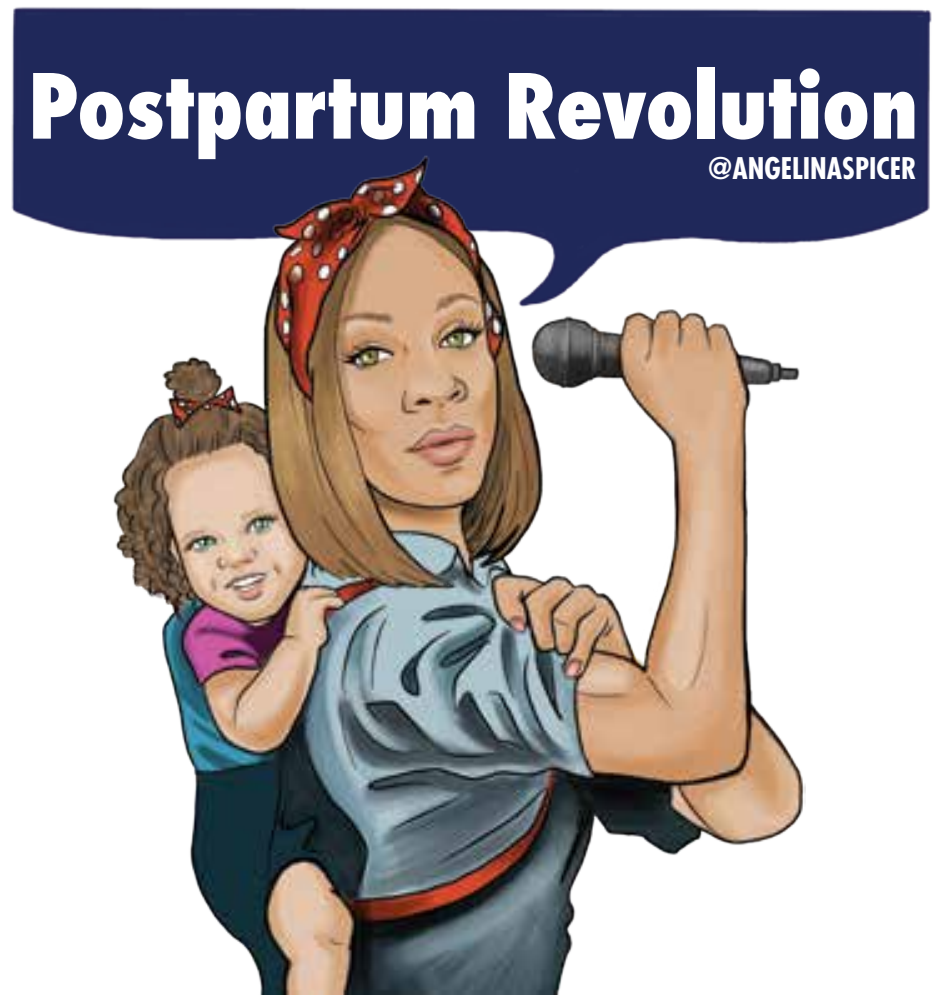

The $34^{\text {th }}$ Annual Gravens Conference on the Environment of Care for High Risk Infants March 3, 4, 10, and 17, 2021: Virtual live March 24 - September 30, 2021: On-demand www.thegravensconference.com

Early Bird Registration through Dec. 31, 2020
Provided by:

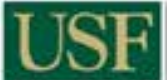

HEALTH

For more information, contact the meeting planner at nrose@usf.edu 